which have penetrated them. So large a field of new observations and new methods of treatment is present here that it would have been easy to take up all the time this evening by going over it alone. This was impossible but I must mention one. At last some use has been found for the appendix, that abortive organ which bas been so much denounced as an entirely superfluous viscus. In certain cases of mucous or membranous colitis which relapse after all treatment apparently because local applications fail to reach the cæcum, the cæcum itself has been opened, an artificial anus has been made, and the large gut has been washed out from end to end. Weir ${ }^{17}$ has proposed that instead of making a new opening into the cæeum the appendix should be found, brought through the abdominal wall, and fixed there. Its end beicg cut off, its lumen is utilised as the cbannel for injections. Of course care must be taken that this little tabe is not ulcerated and that it is permeable. When once the tissues have returned to their normal state this can either be removed in the usual way or, which seems preferable, its minute opening can be closed, itself remaining ready for use again should the condition relapse. Weir, and following him Meyer, ${ }^{18}$ have reported cases in which this method has answered admirably and it certainly appears to be a valuable addition to our operative resources in combating these at present often most unsatisfactory cases. In the one case in which $I$ have used it the proceeding was perfectly easy to carry out and was most successful.

As was evident from the first, a large number of chronic abdominal surgical conditions have been necessarily excluded from consideration. To mention no others, there is a large field for description and discussion in growths peculiar to women, but sufficient has been said, perhaps, to emphasise the proposition with which I started-viz., the necessity for constant observation of chronic cases with a view to the discovery if possible of new symptoms which will throw light upon their earlier stages and which may act as dangersignals, so that effective surgical interference may be carried out whilst there is every reason to expect a successful issue and before dangerous complications have arisen, or at least before they have reached such a stage as to render all our efforts intffectual.

It only remains for me to thank you for the patience with which you have listened to what, I am well aware, has been but a feeble and ineffectual attempt to place this question before you.

\section{PHYSIOLOGICAL OR FUNCTIONAL ALBUMINURIA. ${ }^{1}$}

BY SAMUEL WEST, M.D. OXON.,

PHYSICIAN AND IKCTURER ON PRINCIPLFS AND PRACTICE OF MFDICINE, ST. BARTHULOMEW'S HOSPITAL; CONSULTING PHYSICIAN, ROYAL FREE HOSPITAL, ETC.

THE problem of physiological albuminuria from its clinical side presents itself thus. A patient has albumin in the urine without any sign of organic disease, what is its significance and importance? The difficulty here lies, as it does so often in medicine, in applying general rules to the individual case. The cases of albuminuria fall into two groups according as the cause lies in the kidney itself or not. Where the kidney is not itself the organ at fault the cause may be found in the heart or in abnormal conditions of the circulation, in blood changes or other general conditions, and in each case alike the cause may be obvious or not obvious. In a third group of cases the urine is normal when secreted, the albumin being added to it as it passes from the kidney to the exterior. The following table makes the classification plain :-

$$
\left.\begin{array}{lll}
\text { Cis- or pre-renal } & \begin{array}{l}
\text { Cause obvions-e.g., morbus cordis, fevers, \&c. } \\
\text { Cius not obvious. } \\
\text { No obvious renal disease. } \\
\text { Obvious renal disease. }
\end{array}
\end{array}\right\} \begin{gathered}
\text { Physiological or } \\
\text { Runctional. }
\end{gathered}
$$

Citra- or post-renal-accidental, from urinary or genital passages, \&c.

Physiological albuminuria might, therefore, be described, as this tarle suggests, as albuminuria in which the cause is not obvious. This makes plain the indetinite character of

$$
\begin{aligned}
& \text { 17 Medical Record, August 9th, } 1902 \text { p. } 201 . \\
& 18 \text { Medical News, vol. i., 19j3, p. } 12013 .
\end{aligned}
$$

1 A paper introrucing the discussion at tne Medical Society of Iondon on Dec. 14th, 1903. this group of cases and the difficulties which attend the diagnosis.

The first thing to be done clinically in a case in which albumin is found in the urine is to exclude all accidental contamination-i.e., to make sure that the albumin is not added to the urine after secretion on its way from the kidney. In women such contamination is common from the vagina and uterus but it can easily be eliminated by obtaining a clear catheter specimen. There remain, however, in man and woman alike other accidental sources which are not so easy to exclude-e g., discharges from the urethra, the prostate, the bladder, the ureters, and the pelvis of the kidney. The presence, lor instance, of a small calculus in the pelvis of the kidney may lead by its irritation to the occurrence of albumin, as it does of blood, without other symptoms of its presence. This is especially true of an oxalic acid calculus and even of oxalic acid crystals too. An extreme instance of this kind is the copious albuminuria attending an acute attack of gout, when tor the first day or two the urine may be loaded with albumin which disappears as rapidly as it came when the attack sub.ides. Some of these accidental causes are by no means easy to eliminate. Supposing this to have been correctly done the next step is to exclude all cases where there are obvious lesions in the kidney or morbid conditions elsewhere in the body sufficient to explain the albuminuria-e.g., morbus cordis, fevers, certain conditions of the blood, \&c. This is generally not a difficult matter.

There then remains a group in which no cause can be found to which the albuminuria can be referred. The patient, therefore, is assumed to be healthy and the albuminuria is called physiological or functional. What physiological or functional albuminuria then means is that every care has been taken to find a cause but without success. It does not, however, follow that because a cause has not been found no cause exists and that the albuminuria is but an accident of health. Physiological albuminuria, therefore, is a diagnosis based partly on fact and partly on opinion. The fact is certain. The opinion may be open to question and its value depends upon the care which has been bestowed upon the investigation of the case. It is a pity, therefore, to use a term which seems to beg the very question which bas to be proved. The term "functional" seems to me even more objectionable than "physiological" as being still more indetinite and vague.

Other terms have been suggested such as intermittent, cyclical, postural, and dietetic, but they are all open to objection. I think it would be best to discard the term physiological or functional altogether and to speak of the condition as albuminuria in the apparently healthy. This shows where the difficulty lies, for it is often impossible to be certain that the patient is in fact healthy.

Whether it be called physiological or functional the first inquiry which the name suggests is this: Does albuminuria occur under normal or physiological conditions? It is stated that albumin may be demorstrated in the urine of everyone in some such way as the following. Urine, in which the ordinary tests have failed to show albumin, is concentrated and the re:idue is dissolved and carefully tested. Then a trace, very minute it may be but still sufficient, may be demonstrated by delicate tests. The explanation of this seems obvious. There is a constant shedding of epithelium throughout the urinary passages. If this epithelium be concentrated, collected, and dissolved it will, of course, give the reaction for albumin. After all this question is of academic interest rather than of clinical importance, for no one suggests that albuminuria is ever physiological in the sense that albumin may be demonstrable by the ordinary means in the unconcentrated urine of any and every one.

Statistics showing the frequency of albuminuria in the apparently healthy differ widely. Thus examinations made in the ordinary routine of out-patient practice yield a percentage of 1,2 , or 3 as the case may be. Mure careful examinations made ad hoc raise the percentage several points, it may be to 10. Again, frequent examinations at different times of the day may show albuminuria prerent at one time and not at another. In this way by careful examirations frequently made and with varied delicate tests the percentage may be considerably raised.

For the purpose of the Lettscmian lectures in $1899 \mathrm{I}$ had a long series of observations made by the late Dr. Levison which yielded one of the highest percentages obtained-viz., 42. The analyses of these results are very instructive. First, it may be noted that of healthy perscns nearly 60 
per cent. show not the slightest trace of albumin with any test at all. Of the 40 per cent., in nearly one-half the form of albumin was not serum albumin but nucleo-albumin, and that only in very minute trace. Of the 20 per cent. in which the albumin was serum albumin, in about one-half the trace was so minute that it would have been overlooked in ordinary examination. Thus we arrive at 10 per cent. in which the albumin would have been discovered by the ordinary methods of testing-a percentage which is not far removed from that of ordinary experience. Finally, even in this residue of 10 per cent. the amount was in most cases extremely small. The frequency of albuminuria varies considerably at different ages. Of healthy infants 10 per cent., it is said, present albuminuria and among the somewhat older patients of a children's bospital it has been shown in about 20 per cent. The most abundant statistics are for the period of school life among boys-and after that for young adults-when the percentage may reach as high as 20 or so. Albuminuria stands at its lowest level about the age of 25 years and from that period continues to rise regularly and rapidly with the age.

Functional albuminuria is very often intermittent and varying in amount. It has sometimes been called cyclical but the regularity of its recurrence depends upon the regular recurrence of the cause-such as changes of posture from the recumbent to the erect position, as on getting up in the morning, exercise, diet, digestion, bäthing, exposure to cold, \&c. In most healthy persons, however, none of these conditions is competent to excite albuminuria. There must therefore be some other cause or peculiarity which determines it and careful clinical investigation will in many cases succeed in finding one. It is, of course, dangerous to make a diagnosis of disease upon a single physical sign. But the converse is also true and it is equally dangerous to assume that because no other abnormal sign can be discovered the one physical sign present can be disregarded and the individual pronounced healthy.

I now turn to some of the more special difficulties which arise in practice.

1. The fact that albuminuria is intermittent, occasional, or oyclical is no proof that the kidneys are sound. In a case which is convalescent from acute Bright's disease and presents no albuminuria while confined to bed, the albuminuria may return on getting up or be brought back by very slight causes, such as changes in diet, exposure to cold, \&c. In fact, all the peculiarities of functional albuminuria may be reproduced. Here it must be agreed that the kidneys, though getting well, are not yet sound. Indeed, as convalescence proceeds the causes referred to fail to produce albuminuria and the recovery is regarded as complete. Of course, in most of these cases the albuminuria is associated with the presence of casts and cells, but, as stated, a few cells may be found in the urine of everyone and the real significance of a few hyaline casts is not yet agreed upon. Then, again, in granular kidney the albuminuria may be intermittent and may be absent for long periods of time and the urine contain few, if any, casts or cells.

2. Again, though in functional albuminuria the amount of albumin is tiny or but a small trace, this may be so in granular kidney; while in convalescence from acute nephritis, where convalescence is not complete, a small amount of albuminuria may persist without other symptoms for years. Even in functional albuminuria the amount is not always small. There are cases described, though they are rare, in which the amount was as much as one-half or more.

3. Again, the clinical importance to be attached to the albuminuria varies greatly with the age of the individual. In newly born babies and infants albuminuria without obvious cause is by no means uncommon. The same holds good to some extent of older children. It is common experience that little children are easily upset by improper or impure food which, besides causing intestinal disturbance, rashes such as urticaria or erythema, and even fever, may lead to transient albuminuria. In fact, in young children all the functions are in a condition of instability. The organs and tissues are not seasoned and resistant ; their condition is easily disturbed by slight causes which in older children and in the adult would produce little, if any, effect, On the other hand, the processes of growth and development are active and recovery is rapid and complete in children where in the adult it would be prolonged and often incomplete. Hence the experience in children cannot be applied without considerable qualification to more advanced periods of life.
4. It is between the ages of 15 and 25 years-that is to say, in boys at school and young adolescents that functional albuminuria has been most frequently described. The statistics at these age periods are abundant and they prove that albuminuria is very common in apparently healthy young people. This is the time of rapid sexual development and naturally the albuminuria has been referred to this cause. The two facts are coincident but there is no reason whatever to believe that they are related. The same is true of masturbation; in this case, at any rate, what evidence is forthcoming is strongly opposed to such an explanation.

The boys at school who present what is called functional albuminuria fall into two groups: the one florid, fullblooded, vigorous lads with good or high pulse tension, the other pale, flabby, not well conditioned, and with low arterial tension. The two groups probably present differences in the pathology and prognosis and demand different treatment and management. The significance of these cases can only be determined by watching their subsequent history. Some, the majority, get quite well soon. In some the albuminuria persists for a time but ultimately disappears, in others it is persistent, while in the residue signs of organic kidney mischief ultimately reveal themselves.

Speaking generally functional albuminuria in boys raises serious questions, for though many seem perfectly well and others get quite well still the fact that others do not get well and the difficulty of deciding to which category any individual case belongs introduce factors of uncertainty in forecasting its future. The practical question which is certain to be raised in these cases is this: "Shall the school life of the boy be interfered with?"-a question of very great gravity to the boy and his parents and of great anxiety and responsibility to the medical attendant. Unless there ase special reasons in the particular case-and each case of the kind has to be settled not upon general principles but upon its own merits-careful consideration and the balancing of all the pros and cons generally end in leaving the boy at school under the careful and constant supervision of his medical attendant. The risk has to be run by the boy and fortunately in most cases the risk proves to be slight and the result is satisfactory.

5. In the young adult, up to the age of 25 years or thereabouts, the questions are the same but of still greater importance, as the answer given may involve the loss of an appointment or of the power to effect a life insurance, upon which much may depend. At this age period-i.e., between 20 and 25 years-the frequency of albuminuria stands at its lowest. After the age of 25 or 30 years the frequency of albuminuria rises as age advances and its significance becomes more serious. Its gravity is also more easy to demonstrate, for statistics bearing directly on the question are available from insurance offices. In my Lettsomian lectures I referred to some experience on this point and I may be excused if $I$ reproduce some of the figures $I$ then quoted to prove the increased risk there is in these cases and the necessity of protecting the life offices against them. From the data stated a calculation was made for me by an actuary to show approximately the difference in mortality compared with healthy males.

\begin{tabular}{|c|c|c|c|c|c|}
\hline \multirow{2}{*}{$\underset{\text { attained. }}{\text { Ages }}$} & \multicolumn{2}{|c|}{ Rate of following year. } & \multirow[b]{2}{*}{$\begin{array}{c}\text { Ages } \\
\text { attained. }\end{array}$} & \multicolumn{2}{|c|}{ Rate of following year. } \\
\hline & $\begin{array}{l}\text { Rate from } \\
\text { figures } \\
\text { given. }\end{array}$ & $\begin{array}{l}\text { Rate for } \\
\text { healthy } \\
\text { males. }\end{array}$ & & $\begin{array}{l}\text { Rate from } \\
\text { figures } \\
\text { given. }\end{array}$ & $\begin{array}{l}\text { Rate for } \\
\text { healthy } \\
\text { males. }\end{array}$ \\
\hline 30 & - & - & $50 \frac{x}{2}$ & $3 \cdot 70$ & $1 \cdot 97$ \\
\hline 32 & $1 \cdot 53$ & $1 \cdot 06$ & $52 \frac{1}{2}$ & $2 \cdot 64$ & $2 \cdot 20$ \\
\hline 34 & $3 \cdot 27$ & $1 \cdot 11$ & $54 \frac{1}{2}$ & $3 \cdot 74$ & $2 \cdot 43$ \\
\hline 36 & $3 \cdot 33$ & $1 \cdot 16$ & $56 \frac{1}{2}$ & $7 \cdot 13$ & $2 \cdot 69$ \\
\hline
\end{tabular}

The numbers are, of course, too small to lay any stress upon the actual percentages, but the $f_{i}$ ures : how in a general way how largely the presence of albuminuria raises the mortality as well in those below 40 years of age as in those above. This introduces the question which runs through all the age periods alike but becomes most pressing after the age of 30 years-viz., the existence of latent kidney disease, in other words, the relation of these cases to granular kidney. That this relation must be fairly close, closer than is generally thought, is demonstrated by the diagram which shows the curve of granular kidney and that of albuminuria. to be of the same character. 
In granular kidney it is known that the albumin in the urine may vary greatly in amount; that it is often a mere trace; that it may be absent altogether, even for long periods; that it may not be associated with casts or renal cells; and that in its occasional and varying character it may present all the features attributed to functional albuminuria. Further, granular kidney is a very insidious

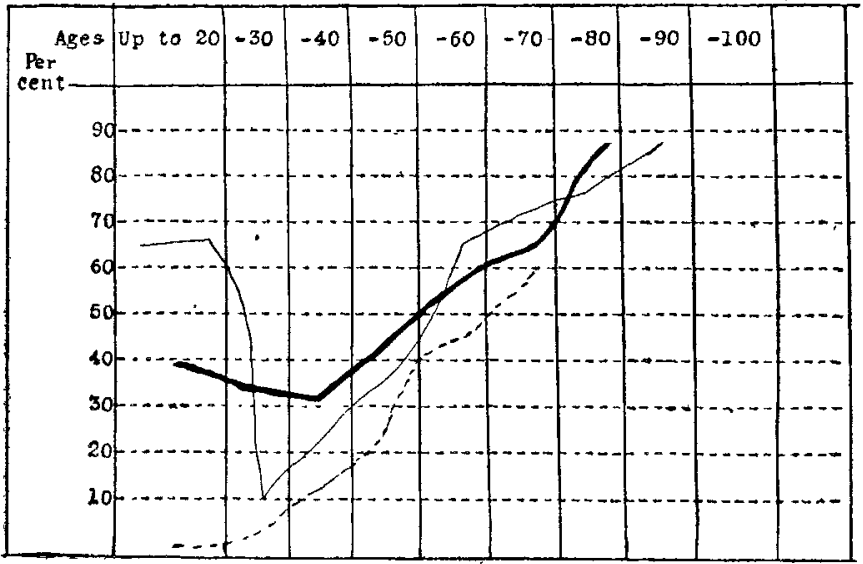

Diagram constructed from collated statistics given by Stirling (light line) and Levison (thick line). The dotted line shows the frequency of granular kidneys in proportion to healthy kidneys. It is drawn in accordance with Mahomed's figures.

disease which may lie latent or be undiscovered for years. It is a disease of long duration and, though the period of greatest mortality is in middle life, it must, running the chronic course it does, have existed for many years previously. In other words, the beginning of granular kidney has to be looked for in quite early life. Thus many cases of advanced or fatal granular kidney occur under the age of 30 years, some are met with under 20 years, and a few even in children. Granular kidney has been even described in infants, but as it is not in accord with our general pathological experiences to believe that advanced fibroid degeneration of any organ can develop in a few weeks these cases in infants probably have a different pathogeny and may be attributed to some intra-uterine or congenital defect of development. Interesting as these cases undoubtedly are they do not 'affect the general poirt at present under consideration-viz., the fact that the commencement of the pathological process which leads ultimately to granular kidney must be referred to early life, even to the period of adolescence or puberty, the very age at which functional albuminuria is most frequently described.

If then there is no age at which granular kidney may not be present or be developing, it is evident that some of the cases of so-called physiological or functional albuminuria may be early cases of granular kidney in which the other signs of that disease are not yet developed, or in which that disease has been either not sought for or even overlooked. It is therefore important that in all cases of functional albuminuria careful search should be made for the signs of granular kidney. Of these signs, besides albuminuria, the most important is arterial thickening (arterial sclerosis). Of arterial thickening there are two well-known forms-atheroma in advanced life and that associated with granular kidney in middle and early life. Besides these two there is no other widespread or general arterial sclerosis recognised. Arterial thickening is not uncommon in young people and it can hardly be due to atheroma. There is, however, in my opinion, one other form of arterial thickening which is met with in the young and lusty. This is probably a physiological hypertrophy. It is seen in athletes and is, I believe, due to a hypertrophy of the muscular coat of the larger arteries similar to that which occurs in the heart. I have scen not a few cases in which this seemed to be the only admissible interpretation. With this exception, however, so far as $\mathbf{I}$ know, arterial thickening in the young must always be looked upon with suspicion. Thickened arteries may suggest granular kidney when albumin is absent and albuminuria may be due to granular kidney when the arteries are not especially thick but the association of the two conditions is very serious.

Thickened arteries are not sufficiently and systematically looked for in the young. If they were they would be more frequently found. In many of the statistics of physiological albuminuria no reference whatever is made to the condition of the arteries and this omission certainly goes some way to vitiate the importance of the figures given. If these considerations be now applied to life insurance we must conclude that albuminuria even in the apparently healthy-i.e., the socalled functional albuminuria-introduces an appreciably increased risk to the life and that this risk increases rapidly with age. The rules which have been laid down are sound and fully justified-viz., to reject all such lives above the age of 40 years, to load them heavily between 30 and 40 years, to make some addition to every case under 30 years, and to wait awhile and to watch the cases in adoles. cence and youth. To which general rules may be added the still more important one to reject at once at every age those cases in which the albuminuria is associated with arterial thickening.

The conclusion of the whole matter may be summed up thus:-

1. Albuminuria may occur as a transitory symptom in persons who, except for this symptom, may be judged to be perfectly healthy, for they appear to be so at the time and remain so.

2. But it may also occur in persons who though they appear at the time to be healthy develop signs of disease subsequently.

3. It is difficult to distinguish at a given time between those who will remain well and those who will not.

4. It is difficult to exclude for certain many of the pathological causes to which the albumin might be due. In other words, though there may be no proof that these causes are present there is equally no proof that they are absent and in some cases the result shows that they were not absent.

5. Speaking generally, the larger the amount of albumin in the urine and the longer it persists the greater the probability of some permanent disease.

6. Continued observation of these cases shows that the so-called physiological albuminuria does very appreciably increase the risk of life and that this risk grows rapidly with every year of age after 30 years.

As the result of the foregoing proposition we are led to the general conclusion that tunctional albuminuria is never, strictly speaking, physiological at all, but that it is, on the contrary, always pathological though not necessarily renal.

Wimpole-street, W.

\section{THE TERMINATION OF A CASE OF RECURRENT MAMMARY CANCER TREATED BY OÜPHORECTOMY.}

\section{BY G. ERNEST HERMAN, M.B., F.R.C.P. LoND., F.R.C.S. ENG.,}

CONSULTING OBSTETRIC PHYSICIAN TO THE LONDON HOSPITAT.

IN THE LANCET of June 11th, 1898, p. 1612, I published a report of a case of recurrent mammary cancer treated by oopphorectomy. The cancer was noticed and removed in October, 1890. At the end of 1896 it had recurred and had invaded the axilla and neck so extensively that removal of the recurrent growths was impossible. In March, 1897, the ovaries were removed. By September of that year none of the recurrent growth could be felt. The patient remained in good health until the autumn of 1901. During the greater part of September, October, and November she had evening pyrexia and perspirations, the cause of which was not found out although repeated careful examinations were made. In January, 1902, she again had evening pyrexia. In February she had an attack of pain in the region of the left kidney so severe as to need the injection of morphia. In March she began to suffer from epigastric pain after taking food. In October Dr. G. O. Tayler of Trowbridge, her medical attendant, discovered an oval, elastic, painless swelling in the line of the sagittal suture, near its anterior end, measuring about one inch by three-quarters of an inch. This increased slowly in size during the rest of her life. The epigastric pain continued and in January, 1903, she began to have dorsal pain. In March attacks of vomiting commenced and her feet became odematous. There was then no albumin in the urine. Later finid was felt in the abdomen. In May a little blood was twice present in the urine. Her breathing became so difficult that she could not lie down. 